\title{
Evolving recursive programs by using adaptive grammar based genetic programming
}

\author{
Man Leung Wong
}

(C) Springer Science + Business Media, LLC 2006

\begin{abstract}
The publisher apologizes for an error that occurred in the above mentioned article. The error appears in the printed version, as well as in the html and pdf version online. Man Leung Wong is the sole author of this article. His affiliation is listed below.
\end{abstract}

The online version of the original article can be found at http://dx.doi.org/10.1007/s10710-005-4805-8. 